\title{
INVESTIGACIÓN HISTÓRICA DE LAS HERNIAS LATERALES DE LA PARED ABDOMINAL*
}

\author{
Dr. Alfredo Moreno-Egea ${ }^{1}$ \\ 1 Unidad de Pared Abdominal. Departamento de Cirugía. Hospital Universitario José María Morales Meseguer. \\ Murcia. España.
}

\begin{abstract}
Historical analysis of the lateral abdominal wall hernias

Objective: To analyze the history of lateral abdominal wall hernias. Method: Review of the literature. Critical analysis of articles, books and monographs published, using key words: "ventral, lateral or semilunar abdominal wall hernia". Results: Undocumented contributions, such as Mancke, Molliére, Reynier, Ferrand or Thèvenot, among others are discovered. Casseri was the first surgeon to illustrate the semilunar line, before Spiegel. Mancke was the first surgeon to use the term lateral ventral hernia. Molliére was the first surgeon to use the term semilunar hernia (not Klinkosch). Thévenot was the first surgeon to use the term Spiegel hernia. Conclusion: The story of the lateral hernias has errors and omissions of important authors. 1) semilunar line was known and illustrated first by Casseri, so we should call Spiegel-Casseri semilunar line; 2) Klinkosch was not the first to use the name of semilunar hernia, but Molliere, who defined them as a different group of ventral; 3) Mancke was first named as the lateral hernias, and Ferrand side who made the first thesis under that title, and 4) the contributions of Reignier and Thévenot, not mentioned in any treatise on surgery are discovered.
\end{abstract}

Key words: Semilunar hernia, lateral ventral hernia, Spiegel, Casseri, Molliére.

\section{Resumen}

Objetivo: Analizar la historia de la hernia lateral de la pared abdominal. Método: Revisión de la literatura. Análisis crítico de los artículos, tesis, libros y monografías publicadas, utilizando como palabras clave: "hernia ventral, lateral o semilunar". Resultados: Se descubren aportaciones no documentadas, como las de Mancke, Molliére, Reynier, Ferrand o Thévenot, entre otros. Casseri fue el primer cirujano en ilustrar la línea semilunar, antes que Spiegel. Mancke, el primero que utilizó el nombre de hernia lateral del abdomen. Molliére fue el primero que usó el término hernia semilunar (no Klinkosch), y Thévenot el primero que usó el término hernia de Spiegel. Conclusión: La historia de las hernias laterales presenta errores y omisiones de autores importantes: 1) la línea semilunar fue conocida e ilustrada primero por Casseri, por lo que deberíamos llamarla línea semilunar de Spiegel-Casseri; 2) Klinkosch no fue el primero en utilizar el nombre de hernia semilunar, sino Molliére, quien las definió como un grupo diferente de las hernias ventrales; 3) Mancke fue el primero que las nombró como hernias laterales y Ferrand quien realizó la primera tesis bajo ese título, y 4) se descubren las aportaciones de Reignier y Thévenot, no mencionadas en ningún tratado de cirugía.

Palabras clave: Hernia semilunar, hernia ventral lateral, Spiegel, Casseri, Molliére.

*Recibido el 21 de julio de 2014 y aceptado para publicación el 27 de agosto de 2014.

El autor no refiere conflictos de interés.

Correspondencia: Dr. Alfredo Moreno-Egea

morenoegeaalfredo@gmail.com 


\section{Introducción}

Las hernias laterales de la pared abdominal (HL) se definen como aquellas que emergen por fuera de la línea alba y de los orificios inguinocrurales. Se las llama también hernias de la línea semilunar, o de Spiegel, denominación que como veremos tras nuestro estudio, es errónea. Son un tipo de hernias poco documentado, y su historia está plagada de interrogantes, y errores, sobre todo en muchas de las referencias que se han ido transmitiendo, sin verificar las citas originales de donde se habían recogido, y en la omisión de muchos cirujanos cuyas aportaciones no han sido recogidas en ningún texto de la especialidad. Este estudio tiene como objetivo analizar la historia de la HL, con los avances metodológicos actuales, corregir este déficit de información, y dar la oportunidad al lector de poder conocer, de una forma fiable, los personajes que en ella han participado.

\section{Método}

Se realiza una revisión exhaustiva de la literatura a través de las bases de datos MEDLINE (PubMed), LILACS (incluyendo SciELO) y la Cochrane Library, utilizando como palabras clave: "hernia ventral, lateral o semilunar". Se realiza un análisis crítico de los artículos, tesis, libros y monografías publicadas. También se realiza una búsqueda complementaria mediante "Google" (Google play), de las bibliotecas de universidades (Padua, Standford, Leipzig, Paris, etc.), y por bibliotecas (Medical Heritage Library). Se estudiaron los artículos obtenidos de cualquier país, por cualquier institución o investigador y en cualquier idioma, sin límite temporal.
Los textos originales que nos han sido remitidos, una vez recuperados se han almacenado en formato $p d f$ para preservar su originalidad.

\section{s. XVII: Primeros conocimientos de la pared abdominal lateral}

En el s. XVII la anatomía descriptiva era una ciencia en auge en algunas prestigiosas universidades europeas. La Universidad de Padua se convirtió en el faro del pensamiento científico moderno guiada por sus grandes anatomistas. Giulius Cesare Casseri (1552-1616), fue asistente personal de su maestro Fabrizio. Su vida estuvo marcada por la enemistad con su jefe, hecho que ensombreció su labor y toda su obra. Su gran obra anatómica no fue nunca publicada en vida, por su continua disputa con Fabrizio, y pasó a manos de sus herederos sin ser conocida en su tiempo (pero fue escrita antes de la llegada de Spiegel al Departamento) (Figura 1) ${ }^{1}$.

Adriaan van der Spiegel (1578-1625), fue nombrado profesor de Anatomía y Cirugía en 1616, después de morir Casseri, y llegó a dirigir los dos departamentos de anatomía y cirugía en 1619, falleció en $1625^{2}$. Su obra fue publicada de forma póstuma en 1627, sin ilustraciones, y en ella se describe en apenas unas frases, la línea semilunar ${ }^{3}$. En 1632, Daniel Rindfleischs, publicó dos tomos: un tomo I que contenía el texto de Spiegel, y un tomo II con las magistrales y educativas láminas anatómicas de Casseri, compradas a sus herederos. Este hecho fue el que facilitó la gran difusión de la obra durante todo el siglo XVIII, pero ensombreció la aportación de Casseri frente a la de su sucesor. La historia debería recordar la contribución de ambos, y para ello proponemos llamar a la línea semilunar como "línea de Spiegel-Casseri" (Tabla 1) ${ }^{4}$.

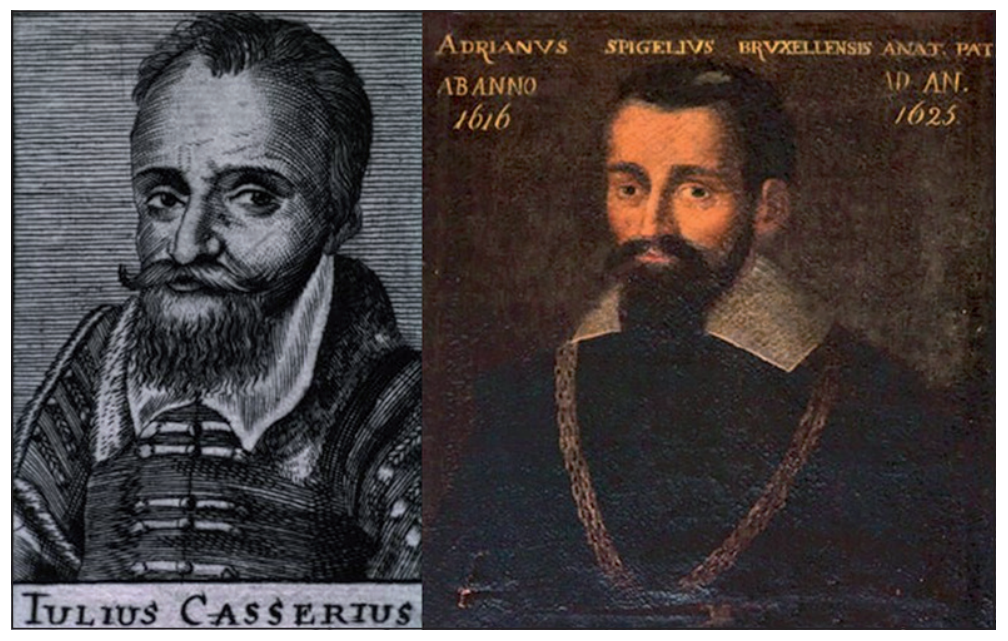

Figura 1. Retrato de Giulius Cesare Casseri (1552-1616) y de Adriaan van der Spieghel (1578-1625), cirujanosanatomistas de la Universidad de Padua. 
Tabla 1. Historia antigua de la hernia lateral: s. XVII-XVIII (1601-1800)

\begin{tabular}{|cll|}
\hline 200 & Galeno (131-201) & $\begin{array}{l}\text { Describe la anatomía de la pared abdominal } \\
\text { Hernia: rotura del peritoneo con estiramiento de fascias y músculos }\end{array}$ \\
1627 & $\begin{array}{l}\text { Giulius Cesare Casseri (1552-1616) } \\
\text { Adriaan van der Spieghel (1578-1625) }\end{array}$ & $\begin{array}{l}\text { Ilustración de la línea semilunar } \\
\text { Descripción de la línea semilunar* }\end{array}$ \\
1742 & Henry-Francois Le Dran (1685-1770) & $\begin{array}{l}\text { Hernia Ventral: diferente a la umbilical y a las de la línea alba. Incluye } \\
\text { un grupo de hernias raras }\end{array}$ \\
1746 & Beatus Ignatius La Chausse (1727-1808) & $\begin{array}{l}\text { Hernias ventrales de los flancos (tipo 3) } \\
\text { Origen Traumático }\end{array}$ \\
\hline 1764 & Joseph T. Klinkosch (1734-1778) & $\begin{array}{l}\text { Hernia Ventral: en la línea semilunar ** (como una posibilidad, sin más } \\
\text { aportación) }\end{array}$ \\
\hline
\end{tabular}

*Publicación póstuma por el médico alemán Rindfleisch (alias Bucretius). ** El análisis documental demuestra que fue Molliére el primero que clasificó a las hernias con el nombre propio de semilunares.

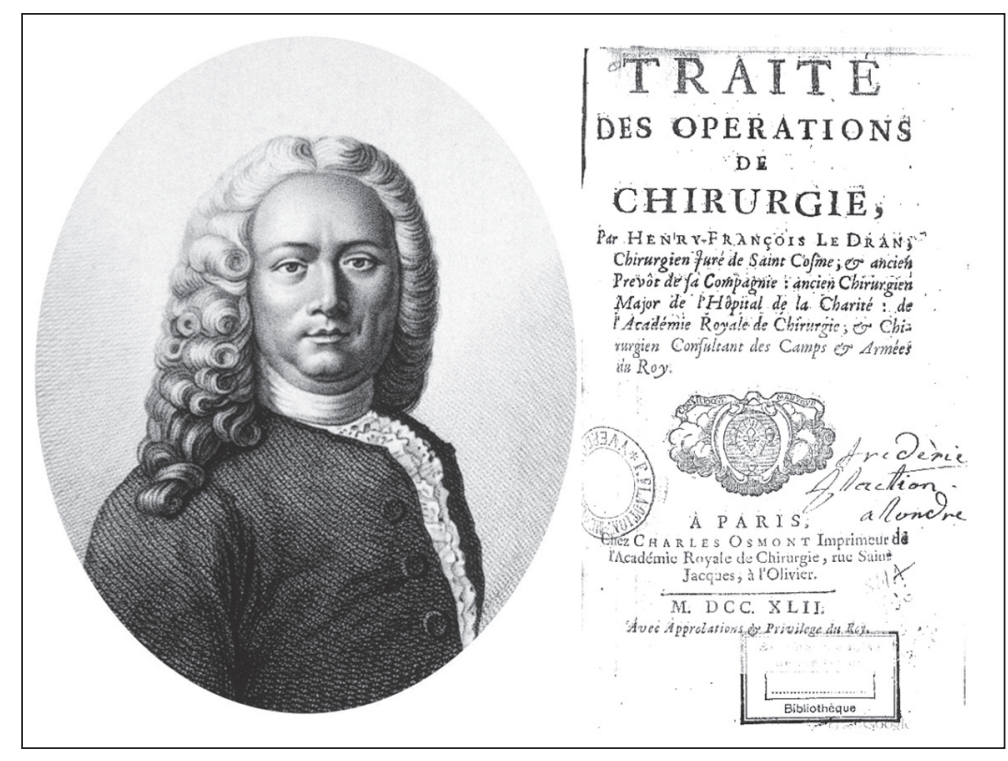

Figura 2. Le Dran y portada de su tratado.

\section{s. XVIII: Historia antigua}

Durante el s. XVIII se cimentaron las bases del conocimiento anatómico de la pared abdominal. Tenemos que situarnos en la escuela quirúrgica de París para continuar esta investigación, cuando los hospitales comenzaron a atender a miles de pacientes como consecuencia de las sucesivas guerras. Por ello, los cirujanos franceses debían conocer muchos tipos de hernias, como consecuencia de heridas, drenaje de abscesos, extracciones de metralla, etc. Le Dran (1685-1770), hijo de cirujano, y reputado profesor de la Charité, publicó en 1742 su tratado, donde considera a las hernias ventrales como una entidad diferente de las umbilicales, epigástricas y de la línea alba, indicando que se forman generalmente a ambos lados de los resistentes músculos rectos del abdomen, y resalta su relación con los embarazos múltiples. Cita además que existe un tipo de hernias ventrales que son más peligrosas porque aparecen externamente o bajo los músculos rectos y pueden pasar inadvertidas, pero nunca utiliza el término lateral ni semilunar en su obra (Figura 2$)^{5}$.

La Chaussee (1727-1808) en 1746, publicó el primer caso originado por un traumatismo abdominal, y distinguió 3 tipos: 1) las que ocurren a través de la línea alba, por arriba y por abajo; 2) las de la región epigástrica lateral y 3) las de la región hipogástrica lateral, causadas por la separación de las fibras de los músculos transversos y oblicuos. Estas hernias ventrales tipo 3 las considera siempre de origen traumático, y remarca su situación sobre el borde externo de los músculos rectos del abdomen. Este tipo se podría corresponder con las HL, pero tam- 


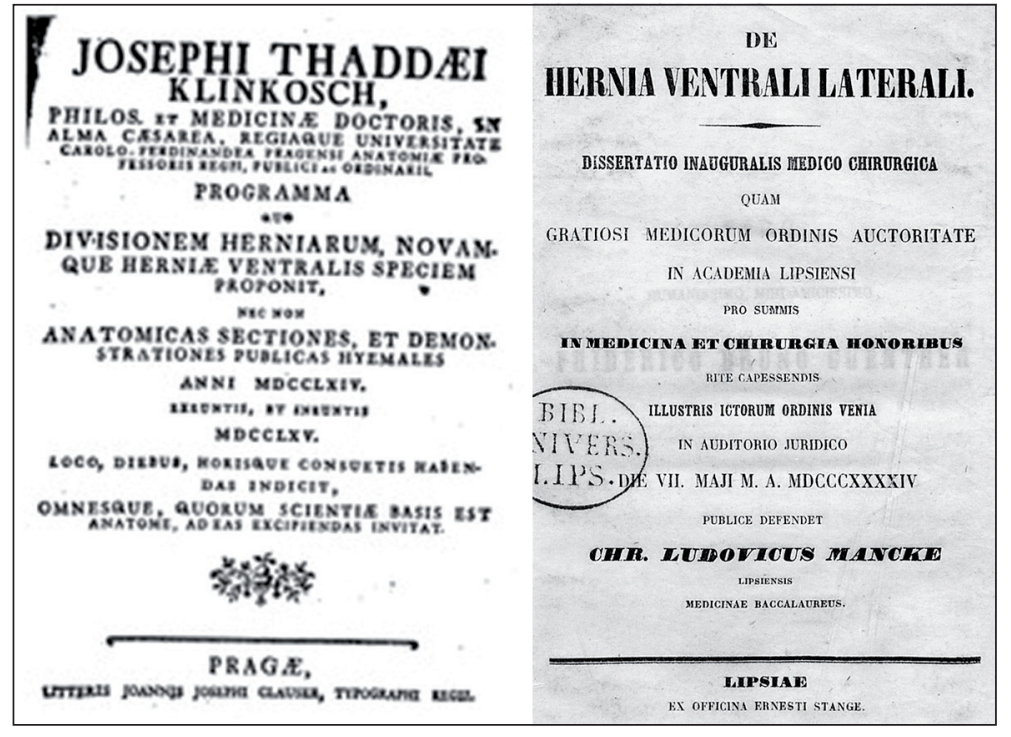

Figura 3. Portadas de los estudios de Klinklosch (Praga) y Mancke (Leipzip).

Tabla 2. Historia clásica de las hernias laterales: s. XIX (1801-1900)

\begin{tabular}{|c|c|c|}
\hline 1804 & Astley Pastor Cooper (1768-1841) & $\begin{array}{l}\text { Teoría vascular } \\
4 \text { causas: espontánea, congénita, trauma y por heridas }\end{array}$ \\
\hline 1844 & Ludovicus Mancke & Tesis: hernia ventral lateral \\
\hline 1877 & Daniel Molliére* & $\begin{array}{l}\text { Clasifica independiente las hernias de la Línea Semi-lunar. Define una } \\
\text { zona de acumulo de casos }\end{array}$ \\
\hline 1878 & Louis-Félix Terrier (1837-1908) & Difícil diagnóstico: hernia intersticial \\
\hline 1879 & Fritz Macrocki & $\begin{array}{l}1^{a} \text { serie publicada de hernias raras ( } 86 \text { casos) } \\
\text { Causas: traumáticas y obesidad extrema }\end{array}$ \\
\hline 1879 & Ambroise Reignier* (1850-19 ¿?) & $\begin{array}{l}1^{\text {a }} \text { Tesis doctoral sobre las hernias ventrales } \\
\text { Factores predisponentes y determinantes } \\
\text { Hernias intraparietales }\end{array}$ \\
\hline 1881 & Jacques-Joseph Ferrand* (1835-1899) & $\begin{array}{l}\text { Tesis: Hernias Laterales del Abdomen } \\
\text { Reúne una serie de } 17 \text { casos ( } 3 \text { nuevos) }\end{array}$ \\
\hline 1890 & Jonathan F.C.H. Macready (1850-1907) & $\begin{array}{l}\text { Descripción de una serie de } 21 \text { casos } \\
\text { Hernia oculta o enmascarada }\end{array}$ \\
\hline
\end{tabular}

*Referencias inéditas en la literatura médico-quirúrgica.

poco utiliza nunca este nombre en su obra. Tiene el mérito de haber considerado otro grupo diferente al que llama de "variedades raras" 6 .

Joseph Thaddaeus Klinkosch (1734-1778), cirujano anatomista de Praga, inició estudios de derecho en 1750, pero los abandonó por los de Medicina, y especialmente por la anatomía. Se licenció en 1761, y llegó a ser profesor de anatomía y más tarde de cirugía. Fue un verdadero hombre de ciencia pero murió joven, a los 44 años. En 1764 publicó un estudio donde describe una hernia ventral sobre la línea semilunar, pero no lo clasificó de forma independiente ni utilizó el término "de Spiegel" (Figura 3) (Tabla 2$)^{7}$.

Durante el s. XVIII, los cirujanos con especial interés en el campo de las hernias, ya no eminentemente anatomistas sino clínicos, van tomando conciencia: I) de la importancia de esta patología (que representa el grueso de la cirugía de la época, junto con el trauma); II) de la necesidad de presentar y debatir sus problemas clínicos pues advierten que algunas hernias ventrales tienen propiedades especí- 
ficas (a pesar de compartir unos mismos principios en cuanto a sintomatología y terapéutica), y III) de la necesidad de replantear una nueva forma de clasificar a las hernias ventrales. A pesar de ello, concluye el siglo con el convencimiento de que estamos ante una entidad clínica diferente, pero sin que nadie se aventure a clasificarlas.

\section{s. XIX: Historia clásica}

El predominio de la cirugía francesa se va desplazando ahora, primero hacia Inglaterra, y después hacia Alemania, como nuevos focos del saber. Sir Astley Paston Cooper, en 1804, fue el primer autor en postular una teoría etiopatogénica, señalando como el sitio de producción de las HL a los forámenes vasculares que existen en la pared abdominal para dar paso a las ramas colaterales de los vasos epigástricos. Cuando estos orificios sean congénitamente mayores de lo normal, y los vasos se hallen rodeados de tejido adiposo, se puede formar una hernia a través de ellos (Teoría vascular). Esta teoría tiene como debilidad el que pocas veces podemos apreciar vasos atravesando el defecto y acompañando al saco herniario. Cooper sigue clasificando a estas hernias con el nombre de ventrales ${ }^{8}$. Más tarde, en Leipzig en 1844, Ludwig Mancke, publica un estudio titulado por primera vez como hernia ventral lateral, donde partiendo de un caso personal detalla muy bien aspectos de la clínica y del tratamiento, pero sin advertir su relación con la línea semilunar (Figura 3) ${ }^{9}$.

De vuelta a París, es el desconocido Daniel Molliére, en 1877, quien presenta a la Sociedad Quirúrgica de París, un caso bajo el nombre ya específico de hernia semi-lunar. Afirma que estas nuevas hernias se presentan en el lugar de menor resistencia, bajo la arcada de Douglas, donde las aponeurosis de inserción de los músculos laterales del abdomen cambian bruscamente de disposición y dejan de formar la vaina posterior al músculo recto, en una línea que une el ombligo con el relieve de la espina iliaca antero-superior. Es por tanto, el primer autor que clasifica y define a estas hernias como un grupo separado del resto de hernias ventrales (y por ello, este mérito no puede seguir siendo atribuido a Klinkosch) (Figura 4) ${ }^{10}$. Sin embargo, este autor sigue siendo desconocido por todos, ya que no ha sido citado en ningún libro de historia, ni tan siquiera en los específicos de cirugía o de la especialidad de pared abdominal ${ }^{11-14}$.

La gran actividad científica de la Sociedad Quirúrgica de París, permite presentar a Louis-Félix Terrier, otro caso clínico bien detallado (1878), de una hernia ventral espontánea, y confirmar la predisposición de estas hernias a situarse en la zona sugerida por Molliére, destacando la dificultad para llegar a un diagnóstico preoperatorio por su situación intersticial (Figura 4) ${ }^{15}$; y a Ambroise Reignier (1789), autor desconocido hasta hoy, la publicación de la primera tesis doctoral, donde se

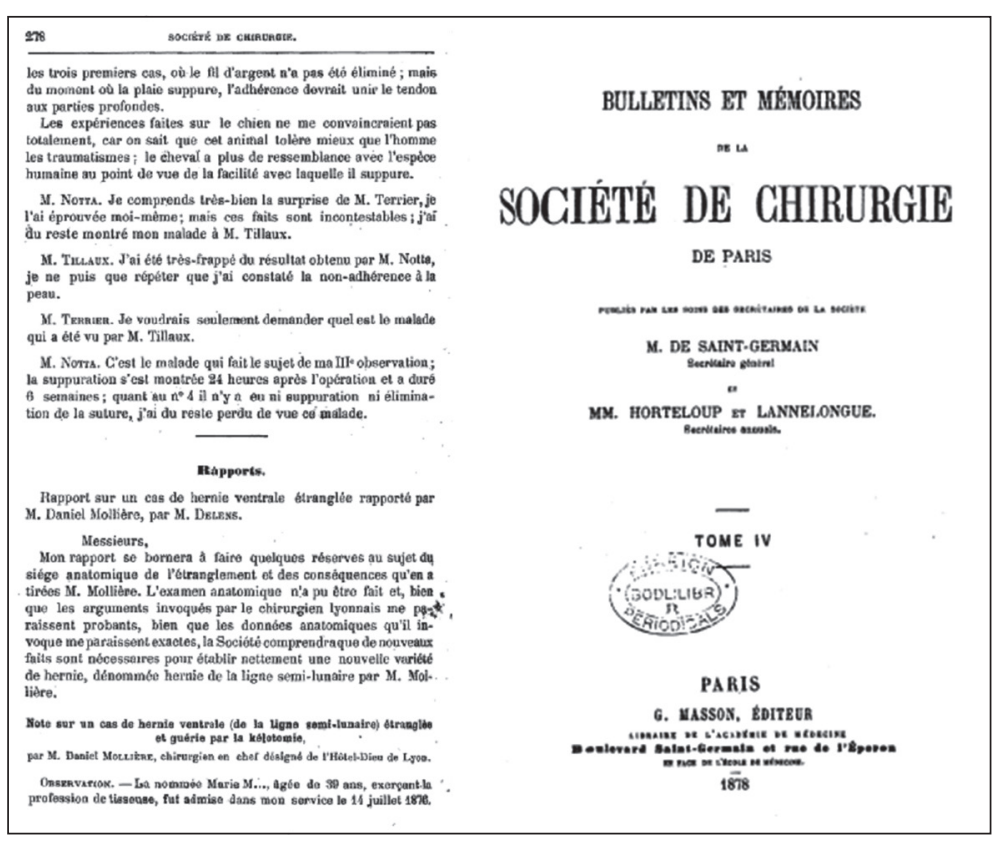

Figura 4. Aportación histórica de Daniel Molliére, y de Louis Terrier, a las hernias laterales del abdomen. 
describe el siguiente mecanismo patogénico: una predisposición anatómica, la presencia de pequeños pelotones de grasa subperitoneal que penetran en los intersticios de entre las fibras aponeuróticas del músculo transverso, en la parte inferior de la línea semilunar, atraviesan los orificios para formar las hernias, y al quedar situadas entre los oblicuos se las denomina hernias intra-parietales ${ }^{16}$. Un año después de la aportación de Terrier, Fritz Mackrocki, reúne 86 casos de HL, ofreciendo una estadística interesante, y destacando como causas frecuentes a los traumatismos y la obesidad extrema. Vuelve a apoyar la hipótesis de que los sitios de origen de estas hernias se relacionan con el paso de los vasos hacia la grasa o "hiatos vasculonerviosos", y aporta un caso traumático tras fractura del hueso iliaco ${ }^{17}$.

En 1881, otro autor descubierto en el curso de esta investigación, Jacques-Joseph Ferrand, diplomado por la Facultad de Medicina de París, interno del Hospital de Bordeaux, y miembro de la Sociedad de Anatomía, publica la que se considera como la primera tesis ya con el nombre de "Hernias Laterales del Abdomen". Este cirujano diferencia en la etiología causas predisponentes (variaciones anatómicas de la línea semilunar), adyuvantes (tumores, quistes, ascitis, etc.), y determinantes (todas las situaciones fisiológicas o actos de fuerza). Da gran importancia a la separación de las fibras aponeuróticas del músculo transverso, hecho que también atribuye a las ramas colaterales de los vasos epigástricos: podría suceder que la hernia se produjese al nivel de los orificios situados cerca del músculo recto, siguiendo de adentro hacia fuera el trayecto de estas arterias, para acabar saliendo bajo la aponeurosis del gran oblicuo al que podrían atravesar entonces (Figura 5) ${ }^{18}$.

En este siglo XIX debemos mencionar dos aportaciones más en el año 1893: la de Macready, que realizó un detallado estudio epidemiológico donde incluye una serie descriptiva de $21 \operatorname{casos}^{19}$; y la de Giordano, que fue el primero en publicar un caso de asociación de hernia de Spiegel con una hernia lumbar ipsilateral ${ }^{20}$. Recordemos que su asociación con otras hernias inguinales, umbilicales y epigástricas ya era conocida por los autores franceses. Como vemos, el s. XIX nos ofrece una comprensión ligada a la concepción fisiopatológica del proceso de las HL, desligándose ya de la rigidez anatómica. La clínica y la cirugía operatoria predominan, y comienzan a referirse las primeras series, experiencias de la que se extraen lecciones de gran interés médicoquirúrgico (Tabla 2).

\section{Primera mitad del s. XX (1901-1950): Historia moderna}

A los conocimientos del viejo continente, donde destacan los primeros estudios sobre cadáveres, se suman ahora las aportaciones nuevas que llegan del otro lado del atlántico. Son los cirujanos americanos quienes recogen en este siglo, el testigo del saber en el campo de la pared abdominal. La aportación fundamental de la primera década todavía nos llega de París, donde en 1907, Thevenot y Gabourd,

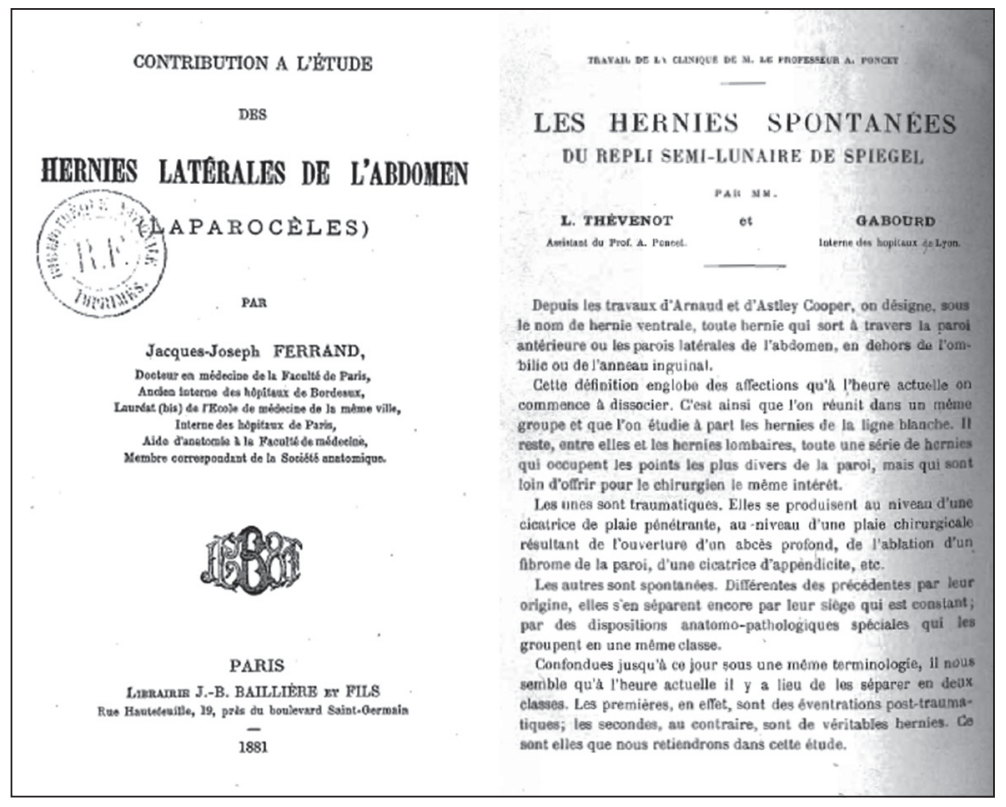

Figura 5. Portada de las tesis olvidadas de Jacques-Joseph Ferrand y de L. Thevenot. 
realizan un interesante trabajo sobre 10 cadáveres, analizando las variantes de la ramificación de los vasos epigástricos. Describen un sitio constante de formación de estas hernias (como había afirmado Molliére) que se corresponde con una predisposición anatómica por fuera del músculo recto, en una línea que une el ombligo con la espina iliaca anterosuperior, y que representa el orificio de entrada de la rama perforante de la epigástrica, de unos $4 \mathrm{~mm}$ de diámetro (Teoría de la perforante vascular). La crítica a esta teoría viene de la experiencia de que existen múltiples sitios de herniación, no un solo punto preciso (Figura 5) ${ }^{21}$. Este autor, al igual que ha ocurrido con Molliére, no ha sido documentado hasta nuestros días. Este es el primer estudio que muestra la importancia de su trabajo. La historia había relegado al olvido grandes cirujanos en los archivos de la biblioteca nacional francesa.

Tres años más tarde (1910), Alfred Stühmer, dermatólogo alemán, publica un estudio donde esquematiza todas las localizaciones de estas hernias, y llama a la más frecuente "punto de Thévenot y Gabourd" (Tabla 3) ${ }^{22}$. Ese mismo año, Wilhelm von Steimker, descubre en el curso de una autopsia, la asociación de una hernia semilunar intraparietal no palpable, con otra inguinal bilateral y otra supravesical $^{23}$. Un año antes (1909), William Bradley
Coley, describe una hernia traumática intersticial erróneamente confundida con otra inguinal asociada, con un retraso diagnóstico de 1 año. El autor destaca la importancia de una cuidadosa exploración física para poder palpar hernias ocultas ${ }^{24}$.

Vingtrinier Barthélemy en 1919, publica otra teoría original, en la que considera que: cuando la vaina posterior del músculo recto no es adherente, no está reforzada por los ligamentos de Hesselbach y de Henle, y la arcada de Douglas es más alta, en el ángulo de dicha arcada con el borde del recto se produce un nicho por donde atraviesan las hernias ${ }^{25}$. A. Augé y René Simon, en 1921, coleccionan 58 casos y destacan que la presión intra-abdominal es fundamental como agente causa ${ }^{26}$. Un año después, Jackson K. Holloway, llama la atención sobre el hecho de que la línea de Spiegel tiene una anchura variable según cada individuo, no es fija geométricamente, y propone hablar de "área semilunar". Recoge 17 casos de la literatura norteamericana y afirma que sólo la cirugía ofrece la esperanza de cura permanente ${ }^{27}$. Un año más tarde (1923), Frank $\mathrm{S}$. Mathews, plantea diferenciar las hernias directas (aquellas que se sitúan por dentro de los vasos epigástricos) en dos subtipos, unas que llama "hernias del tendón conjunto" y otras de la línea semilunar ${ }^{28}$.

S. Y. Koljubakin en 1925, realiza un estudio

Tabla 3. Historia actual de las hernias laterales: primera mitad del s. XX (1901-1950)

\begin{tabular}{|c|c|c|}
\hline 1907 & L. Thévenot & $\begin{array}{l}1^{\circ} \text { que utiliza el nombre de Hernia de Spiegel } \\
\text { Teoría de la perforante vascular } \\
\text { Punto de Trevenot y Gabourd }\end{array}$ \\
\hline 1909 & William B. Coley & Hernia interparietal en el punto de McBurney \\
\hline 1910 & Alfred Stühmer & Teoría de las lagunas congénitas músculo aponeuróticas \\
\hline 1919 & Vingtrinier Barthélemy & $\begin{array}{l}\text { Teoría del nicho (defecto en la vaina posterior del recto por falta de refuerzo del liga- } \\
\text { mento de Henle y Hesselbach) }\end{array}$ \\
\hline 1921 & A. Augé y René Simón & $\begin{array}{l}\text { Teoría del incremento de la presión intraabdominal } \\
\text { Serie de } 58 \text { casos }\end{array}$ \\
\hline 1922 & Jackson K. Holloway & $\begin{array}{l}1^{a} \text { revisión de la historia americana ( } 17 \text { casos) } \\
\text { La línea de Spiegel no es una línea (anchura variable) }\end{array}$ \\
\hline 1923 & Frank S. Mathews & Hernia a través del tendón conjunto (directa o baja) \\
\hline 1925 & S. Y. Koljubakin & $\begin{array}{l}\text { Estudio sobre cadáver }(\mathrm{n}=30) \\
\text { Apoya la teoría de Cooper }\end{array}$ \\
\hline 1927 & Alexius McGlannan & Descripción del mecanismo patogénico \\
\hline 1932 & Mac Neal & Teoría de la fascia transversalis \\
\hline 1942 & Louis P. River & Reúne 116 casos de hernias espontáneas ( 4 americanos) \\
\hline 1944 & L. M. Zimmerman & Teoría músculo-aponeurótica \\
\hline 1948 & J. B. Stenbuck & Hernia abdominal directa (tipo 2 o baja) \\
\hline 1949 & Raymond C. Read & Defensor de la teoría de Zimmerman \\
\hline
\end{tabular}


basado en 30 disecciones, donde analiza las ramas de la arteria epigástrica inferior en relación con la aponeurosis del músculo transverso y vaina del músculo recto. Muestra que la línea de Spiegel presenta perforaciones vasculares en el 50\% de las ocasiones y que se localizan donde había establecido previamente Molliére, siendo el tamaño de dichos orificios de entre 4-18 $\mathrm{mm}$. Ofrece una interpretación de la línea de Spiegel como: aquella entre la porción muscular y tendinosa del músculo transverso, desde el nivel de la $9-10^{\mathrm{a}}$ costilla a un punto $2 \mathrm{~cm}$ lateral a la espina del pubis, con una convexidad hacia afuera y alcanza un punto medial a $5 \mathrm{~cm}$ de la espina ilíaca anterior del íleon, en la línea que llega al ombligo ${ }^{29}$. Alexius McGlannan en 1927, describe una secuencia patogénica mediante la cual, entre las capas profundas de la pared abdominal existen depósitos de grasa preperitoneal que pueden penetrar por los orificios de la aponeurosis acompañando a los vasos. Cuando la grasa aumenta forma lipomas que gradualmente con los movimientos musculares del músculo oblicuo actúan de tracción y se acompañan de un proceso peritoneal creando un verdadero saco herniario. Por este mecanismo la hernia es intersticial, pues la firme aponeurosis del músculo oblicuo externo no es perforada ${ }^{30}$. En 1932 Mac Nealy y Lichtenstein, añaden una teoría etiopatogénica más, al considerar a la fascia transversalis como el verdadero elemento de contención de la pared abdominal, indicando que cualquier defecto, sea congénito, traumático o quirúrgico sobre esta fascia, es la verdadera causa de la $\mathrm{HL}^{31}$. En 1942 Louis P. River, reúne 116 casos de hernias espontáneas, 4 de ellas de la literatura norte americana. Remarca la posibilidad de una susceptibilidad congénita, el carácter enmascarado al ser interparietal con una alta posibilidad de estrangulación, y el ser causa de dolor abdominal no filiado ${ }^{32}$. De todas las teorías etiopatogénicas propuestas, es la de Zimmerman en 1944, la más creíble. La 1lamada teoría músculo-aponeurótica está basada en un estudio sobre 250 cadáveres, donde en el 21,8\% de las veces existen bandas musculares interdigitales entre zonas aponeuróticas débiles en el músculo transverso y oblicuo interno del abdomen, lo que conlleva una debilidad, y cuando existe un defecto congénito se convierten en sitios potenciales de posibles hernias ${ }^{33}$. Stenbuck, en 1948, propone clasificar a las hernias de localización baja como "hernias abdominales directas" 34 . En 1949, Raymond C. Read, reporta un caso que imitaba a la perfección el cuadro clínico de una apendicitis aguda y advierte de la necesidad de recordar esta posibilidad cuando se presente un paciente con dolor abdominal de oscura naturaleza (Tabla 3$)^{35}$. Con este autor llegamos a la mitad de la década y ponemos fin a nuestra investigación. Ahora podemos ya definir algunos aspectos relevantes de estas hernias: I) la propuesta etiológica más verosímil parece ser la de los defectos musculoaponeuróticos; II) el término más adecuado para clasificar estas hernias debe ser el de "lateral"; III) la línea semilunar (de Spiegel-Casseri) representa el borde externo del músculo recto y se extiende desde el pubis al $9^{\circ}$ cartílago costal; IV) la "fascia semilunar" o de Spiegel-Casseri, representa la región donde se fusionan el borde lateral del músculo recto y las aponeurosis de los músculos oblicuo interno y transverso del abdomen. Si esta fusión es defectuosa este espacio es una zona débil.

\section{Conclusiones}

Este estudio refiere que: 1) la línea semilunar, desde un punto de vista histórico, fue conocida e ilustrada primero por Casseri, por lo que deberíamos llamarla línea semilunar de Spiegel-Casseri; 2) Klinkosch no fue el primer cirujano en utilizar el nombre de hernia semilunar, sino Molliére, quien las definió y clasificó como un grupo diferente al resto de hernias ventrales; 3) Mancke fue el primer cirujano que las nombró como hernias laterales, y Ferrand quien realizó la primera tesis bajo este título; y 4) se descubren las aportaciones olvidadas de Reignier y Thévenot, no mencionadas en ningún tratado de historia de la cirugía (ni más específicamente en textos de anatomía o de hernias).

\section{Agradecimientos}

A Doña Teresa González Latorre, documentalista de la biblioteca del Hospital José María Morales Meseguer, de Murcia. Gracias por su apoyo (sobre todo con el Francés y Latín), dedicación y consejo.

\section{Referencias}

1. Riva A. Iulius Casserius (1552-1616): The self-made anatomist of Padua's golden age. Anat Record. 2001; 265:168-75.

2. Spiegel A. De humani corporis fabrica libri decem. Venetiis: Apud Euangelistam Deuchinum; 1627.

3. Spiegel A. De humani corporis fabrica libri decem, tabulis XCIIX aeri incisis elegantissimis, nec ante hac visis exornati. Daniel Bucretius. Serenissimo Ioanni Cornelio Venetiarum duci dicati: opus posthumum. Venetiis: Francofurti Merianus; 1632.

4. Casseri G. Iulii Casserii Placentini Tabulae anatomicae LXXIIX. Venetiis: Durchinum; 1627.

5. Le Dran HF. Traité des operations de Chirurgie. Paris: Osmont; 1742. 
6. La Chausse BI. Dissertatio chirurgica de hernia ventrali. Naples: Halleri Disput; 1746.

7. Klinkosch JT. Programma quo divisionem herniarium. Novumque herniae ventralis specium proponit. Praga: Joan Joseph Clauser; 1764.

8. Cooper AP. The anatomy and surgical treatment of inguinal and congenital hernia. On hernia. London: CT Cox; 1804.

9. Mancke L. De hernia ventrali laterali: Dissertatio inauguralis Medico Chirurgica. Leipzip: Stange; 1844.

10. Molliére D. Hernie ventral de la ligne semi-lunaire guérie par la kélotomie. Bull Soc Chirur. (Paris) 1877; 3:278-84

11. Laín Entralgo P. Historia Universal de la Medicina. Barcelona: Salvat; 1973.

12. Billings JS. The history and the literature of surgery. Philadelphia: Lea Brothers; 1895.

13. Rutkow IM. The History of Surgery in the United States, 1775-1900. San Francisco: Norman Publishing; 1988.

14. Stoppa R. Hernia Healers: an illustrated history. France: Amette; 1998.

15. Terrier, Louis-Félix. Hernie ventral étranglée. Bull Mém de la Soc Chirur. (Paris) 1878;4:361-71.

16. Reignier A. Essai sur les hernies ventrales. Thése de Paris; 1879.

17. Mackrocki F. Beitrag zur pathologie der Bauchdeckenbrüche mit einschluss der sogenannten lumbalhernien. Strasbourg; 1879.

18. Ferrand J-J. Contribution a l'étude des hernies laterals de l'abdomen (laparocéles). Paris: J-B. Bailliére et Fils; 1881.

19. McCready JFChH. Treatise on ruptures. Philadelphia: Robert Purdon; 1893.

20. Giordano D. Contribution allo studio e cura del laparocele. Riforma Medica 1893;30-5.

21. Thevenot L, Gabourd A. Les hernies spontanées du repli semi-lunaire de Spiegel. Rev Chirur. (Paris) 1907;35: 568-85.

22. Stühmer A. Ueber die hernien der bauchwand seitlich der mittellinie unter besonderer Berücksichtigung der Hernien der Linea semilunaris (Spiegelii). Bruns Beitr. Z. Klin. Chirur. 1910; 66:113-35.

23. von Steimker W. Zwel seltenere hernien (hernie supravesicalis externa und hernia ventralis lateralis). Bruns Beitr. Z Klin Chirur. 1913;82:617-50.

24. Coley WB. Interparietal ventral hernia at McBurney's point. Ann Surg. 1909;2:246-9.

25. Barthélemy V. Lateral abdominal hernias. Bull Mém Soc Chirur. (Paris) 1919;45:1313-9.

26. Augé A, Simon R. Contribution ça l'étude des hernies de la ligne semi-lunaire de Spigel. Rev. Chirur. (Paris). 1921;59:299-316.

27. Holloway J. Spontaneous lateral hernias. Ann Surg. 1922;75:677-85.

28. Mathews F. Hernia through the conjoined tendon, or hernia of the linea semilunaris. Ann Surg. 1923;78:3004.

29. Koljubakin SL. Herniae linea Spigelii. Arch fur Klin Chirur. 1925;136:739.

30. McGlannan A. Lateral ventral hernia. Ann Surg. 1927; 85:284-7.

31. Mc Nealy RW. Post-operative hernia: considerations of etiological factors. Am J Surg. 1932;18:90-6.

32. River L. Spigelian hernia: spontaneous lateral ventral hernia through semilunar line. Ann Surg. 1942;116:4059.

33. Zimmerman LM. Ventral hernia due to normal banding of the abdominal muscles. Surg Gynecol Obstet. 1944;78:535-40.

34. Stenbuck JB. Direct abdominal hernia. New York J Med. 1948;48:2404-5.

35. Read Reamond. Observations on the etiology of Spigelian hernia. Ann Surg. 1960;152:1004-9. 